\title{
Septicaemia caused by Neisseria flavescens
}

\author{
PAUL T. WERTLAKE ${ }^{1}$ AND TEMPLE W. WILLIAMS Jr ${ }^{2}$ \\ From the U.S. Department of Health, Education and Welfare, Public Health Service, National \\ Institutes of Health, Clinical Pathology Department, Clinical Center and Laboratory of \\ Clinical Investigations, National Institute of Allergy and Infectious Disease, \\ Bethesda, Maryland, U.S.A.
}

SYNOPSIS Septicaemia caused by $N$. flavescens is reported for the first time. The septicaemia developed following dental surgery, and the clinical picture was indistinguishable from that of chronic meningococcaemia. The skin lesions resembled the cutaneous manifestations of gonococcaemia. The patient responded to treatment with sulphonamides and penicillin with no relapse over 18 months of follow-up.

Neisseria flavescens is a member of the chromogenic, usually non-pathogenic group of neisseria. As such, it is ordinarily a saprophyte of the mouth, nasopharynx, and upper respiratory tree. Whereas other members of the chromogenic neisseria have been reported as the causative agents of endocarditis, and $N$. flavescens has been implicated as the causative agent in meningitis, it has not been previously reported as a cause of bacteraemia. The following report presents a case of $N$. flavescens septicaemia resembling meningococcaemia.

\section{CASE REPORT}

The patient (CC No. 05-15-81), a 20-year-old white woman, was well until seven days after dental surgery (endodontics) when she developed chills, fever, headache, nausea, and vomiting. The febrile illness lasted only six to 12 hours, and she was treated with antiemetics but no antibiotics. During the following 14 days, however, she had three similar bouts of transient fever with chills, headache, myalgia, arthralgias, nausea, and occasional vomiting. The third such episode was accompanied by a rash over the extremities, and local heat and erythema with pain and tenderness in two metacarpophalangeal joints of the right hand. The fever was transient, lasting only six to eight hours, but the rash persisted.

On the fourteenth day following the onset of symptoms the patient was admitted to the Clinical Center, and

'Present Address: The Clinical Laboratory Medical Group, 679 S. Westlake Ave, Los Angeles, Calif. 90057

'Present Address: Baylor University Medical School, Houston, Texas 77025

Received for publication 25 October 1967. shortly after admission developed chills and fever $\left(40^{\circ} \mathrm{C}\right)$. She specifically denied recent insect bites, drug ingestion, or consumption of contaminated foods. She denied sexual contacts, exposure to sick children or adults, and there was no history of surgical or gynaecological manipulations other than the dental procedure one week before the onset of the illness.

Physical examination showed a white woman appearing acutely ill rather than chronically ill. Temperature was $40^{\circ} \mathrm{C}$, and the pulse rate was $110 / \mathrm{min}$. There were numerous erythematous maculo-papular, papulo-nodular and pustular skin lesions over the extensor surfaces of the distal surfaces of the limbs, and a smaller number over the trunk, back, and buccal mucosa. Several lesions on the extremities had necrotic centres.

The right third metacarpophalangeal joint was red, hot, swollen and tender. Large joints, including knees and elbows, were normal. There were no lesions visible in the ocular fundi. The teeth and gums were in good repair although there was heavy tartar on the clinical crown. There was no sign of infection at the site of the recent surgery. Heart sounds were normal with no murmurs. Liver and spleen were not palpable. Pelvic examination was within normal limits. The neurological examination was unremarkable.

Laboratory data at the time of admission demonstrated 12,700 leucocytes per $\mathrm{cu} \mathrm{mm}$ with a shift to the left. Haemoglobin, platelet count, urine analysis, blood urea nitrogen, blood glucose, serum sodium, potassium, and chloride concentrations and carbon dioxide content were normal. Alkaline phosphatase, serum glutamic oxalacetic and pyruvic transaminase, thymol turbidity, total serum protein, and serum protein electrophoresis values were normal.

Serological studies employing specific agglutinins did not demonstrate any significant increase of agglutinins for Salmonella group A. B, C, D, typhoid H, Proteus OX19, and Brucella abortus. The heterophile antibody 
and antistreptolysin $O$ titres were not significantly raised. The C-reactive protein test was strongly positive. Two lupus erythematosus cell preparations and the bentonite flocculation test were negative. Complement-fixation tests for fungi as well as skin tests for fungi and an intermediate strength PPD test were negative. The electrocardiogram and radiographs of the chest and abdomen were normal. $X$-ray examination of the hands revealed a soft tissue swelling about the third metacarpophalangeal joint on the right.

Lumbar puncture showed clear cerebrospinal fluid under an opening pressure of $140 \mathrm{~mm} \mathrm{H}_{2} \mathrm{O}$. The cerebrospinal fluid contained 2 lymphocytes $/ \mathrm{mm}^{3}, 75 \%$ glucose, and $26 \mathrm{mg} \%$ protein. Culture was negative.

A swab from the os of the cervix cultured gamma streptococci, Proteus sp. and Staphylococcus albus. A throat swab yielded alpha streptococci, gamma streptococci, haemolytic Staphylococcus aureus, deoxyribonuclease positive, and a Neisseria sp. which was discarded without additional identification. Culture of urine was negative and two stools were negative for pathogenic bacteria.

Blood cultures, taken hourly for four hours after admission, all yielded a Gram-negative diplococcus. A stained smear of material from a pustular skin lesion revealed Gram-negative cocci, although cultures were negative.

The organism isolated from the blood cultures grew at $22^{\circ} \mathrm{C}$ and $37^{\circ} \mathrm{C}$ on nutrient media in atmospheric conditions as well as in an environment of increased $\mathrm{CO}_{2}$. Growth was obtained from all five blood cultures each of which consisted of approximately $12 \mathrm{ml}$ of blood inoculated into four bottles with the following nutrient media: trypticase soy broth, trypticase soy broth with $0.7 \%$ mucin, brain-heart infusion with para-aminobenzoic acid, and thioglycollate (Hochstein, Kirkham, and Young, 1965). The colonies showed golden yellow pigment formation on Leoffler's serum media. The organism was oxidase positive and failed to produce acid from sugars. Agglutination occurred with a polyvalent neisseria antiserum and types A, B, C, D antisera; however, the quelling reaction was negative indicating a nonspecific reaction consistent with cross-reactivity of a chromogenic neisseria. The organism isolated from each of the four positive blood cultures gave a 1:160 titre with $N$. flavescens antiserum, a positive titre with this antiserum being 1:20 or greater. These characteristics identified the organism isolated from the blood cultures as Neisseria flavescens.

After a provisional diagnosis of meningococcaemia treatment was initiated using procaine penicillin, $1,200,000$ units intramuscularly every six hours, and sulphisoxazole $4.0 \mathrm{~g}$ initially and then $2.0 \mathrm{~g}$ orally every six hours. There was rapid recovery with this therapy. Penicillin was stopped after five days, and sulphisoxazole continued for a total of two weeks. The patient has remained well during 18 months of observation after treatment.

\section{DISCUSSION}

N. Alavescens was first described (Branham, 1930) as an isolate from the cerebrospinal fluid of 14 cases of meningitis occurring during a single epidemic in Chicago in 1928. Subsequently this organism has $\overrightarrow{\vec{F}}$ been reported as the aetiological agent in four other? cases of meningitis (Prentice, 1957; Noguchi, $\frac{\mathrm{C}}{0}$ Nachum, and Lawrence, 1963; Ruiz Sanchez, F., Ruiz Sanchez, A., Negrete, and Rodriguez, 1963). Except for the present case no other reports of septicaemia caused by this agent are known.

The chromogenic neisseria include $N$. flavescens, $\vec{\circ}$ $N$. subflava, N. flava, N. perflava, and $N$. caviae. $\overrightarrow{\vec{H}}$ The habitat of all but $N$. caviae is the mucous mem- $\vec{\omega}$ branes of the respiratory tract of man. The habitat of $N$. caviae is the pharynx of animals. The chromogenicity of these organisms is characteristic on Leoffler's serum medium. Greenish-yellow chromogenesis is typical of the chromogenic neisseria occurring in man. Differentiation of the variouso chromogenic neisseria may be made by sugar? fermentation. $N$. flavescens produces no acid from $\vec{C}$ glucose, fructose, maltose, or sucrose. The other chromogenic neisseria of man produce acid from $\vec{\varphi}$ fructose and $N$. flava acid from sucrose. The organism recovered in the present case was distinguished from $N$. meningitidis by its pigment production and failure to produce acid from glucose or maltose. $N$. meningitidis colonies are non-pigmented and produce acid from both glucose and maltose fermentation.

The clinical picture in the present case resembled $\vec{O}$ chronic meningococcaemia (Benoit, 1963). Chills, $Э$ fever, skin rash, arthralgias and arthritis, headache, and a history of dental work were present. Fever was intermittent and the patient was essentially응 well between the episodes of fever. The skin rash? was polymorphous with maculo-papular, nodular 3 . and pustular lesions, and the patient reported that her rash resembled insect bites as is often the case 3 in chronic meningococcaemia. The rash appeared in the characteristic 'shower' on the extensor surface? of the extremities, the trunk and mucous membranes being relatively spared. The skin lesions were also indistinguishable from those of gonococcaemia $N$ (Abu-Nassar, Hill, Fred, and Yow, 1963).

The severity of arthralgias and myalgias paralleled the fever curve and arthritis did not develop until $\sigma$ the third bout of fever at the time of development of the skin rash. The clinical features of local pain, tenderness, swelling, redness, and heat are those of any infectious arthritis, but the transient nature with involvement of smaller joints has been reported (Pinals and Ropes, 1964) to be a frequent occur- $\frac{\mathbb{D}}{\mathbb{D}}$ rence in meningococcal arthritis.

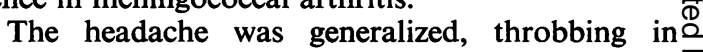
nature and most severe during the height of the fever. Although this organism, like the meningo- 8 
coccus, tends to localize in the meninges, there was no evidence of infection of this site in this patient.

The therapy was that of any neisserial infection with a sulphonamide as the primary agent, and penicillin was added to cover the possibility of sulphonamide resistance or a gonococcal aetiology. The response was as prompt as that usually seen with successful treatment of the meningococcal and gonococcal infections, including chronic meningococcaemia.

\section{REFERENCES}

Abu-Nassar, H., Hill, N., Fred, H. L., and Yow, E. M. (1963). Arch. intern. Med., 112, 731.

Benoit, F. L. (1963). Amer. J. Med., 35, 103.

Branham, S. E. (1930). Publ. Hlth Rep. (Wash.), 45, 845.

Hochstein, H. D., Kirkham, W. R., and Young, V. M. (1965). New Engl. J. Med., 273, 468.

Pinals, R. S., and Ropes, M. W. (1964). Arthr. and Rheum., 7, 241.

Noguchi, T. T., Nachum, R., and Lawrence, C. A. (1963). Med. Arts Sci., 17, 11.

Prentice, A. W. (1957). Lancet, 1, 613.

Ruiz Sanchez, F., Ruiz Sanchez, A., Negrete, D., and Rodriguez, L. (1963). Medicina (Méx.), 43, 25. 